\title{
Ulam Type Stability for a Coupled System of Boundary Value Problems of Nonlinear Fractional Differential Equations
}

\author{
Aziz Khan, ${ }^{1}$ Kamal Shah, ${ }^{2}$ Yongjin $\mathrm{Li}^{3}{ }^{3}$ and Tahir Saeed Khan ${ }^{1}$ \\ ${ }^{1}$ Department of Mathematics, University of Peshawar, Peshawar, Khyber Pakhtunkhwa, Pakistan \\ ${ }^{2}$ Department of Mathematics, University of Malakand, Chakdara Dir (L), Khyber Pakhtunkhwa, Pakistan \\ ${ }^{3}$ Department of Mathematics, Sun Yat-sen University, Guangzhou, China
}

Correspondence should be addressed to Yongjin Li; stslyj@mail.sysu.edu.cn

Received 11 May 2017; Accepted 5 September 2017; Published 11 October 2017

Academic Editor: Hua Su

Copyright (C) 2017 Aziz Khan et al. This is an open access article distributed under the Creative Commons Attribution License, which permits unrestricted use, distribution, and reproduction in any medium, provided the original work is properly cited.

\begin{abstract}
We discuss existence, uniqueness, and Hyers-Ulam stability of solutions for coupled nonlinear fractional order differential equations (FODEs) with boundary conditions. Using generalized metric space, we obtain some relaxed conditions for uniqueness of positive solutions for the mentioned problem by using Perov's fixed point theorem. Moreover, necessary and sufficient conditions are obtained for existence of at least one solution by Leray-Schauder-type fixed point theorem. Further, we also develop some conditions for Hyers-Ulam stability. To demonstrate our main result, we provide a proper example.
\end{abstract}

\section{Introduction}

In last few decades, FODEs become area of interest for the researcher because of high quality accuracy and usability in various fields of science and technology. A lot of physical and natural phenomena can be modeled through FODEs which provide better result than integer order differential equations. Due to this, FODEs are regarded as a special tool for molding. Numerous applications of FODEs can be studied in various disciplines like chemical technology, viscoelasticity, industrial robotics, mathematical economy, turbulent filtration in porous media, fractals theory, ecology, economics, plasma physics, metallurgy, electromagnetic theory, biology, signal and image processing, control theory, electric technology, chemical reaction design, potential theory, radio physics, aerodynamics, pharmacokinetics, and so on; further details are available in literature [1-7]. In last few decades, the existence theory has been given great attention by the researchers. In the concerned theory, they studied existence, uniqueness, and multiplicity of solutions by using different techniques of nonlinear analysis. Therefore, theory on existence and uniqueness of solutions to nonlinear FODEs has been explored very well; see [8-12]. Systems of
FODEs have been considered in large numbers of research articles, because most of physical, biological, and chemical phenomena can be modeled in the form of systems of FODEs. For example, Su [13] studied existence of solutions for coupled system of fractional differential equations with two-point boundary value problems given as

$$
\begin{aligned}
D^{\theta_{1}} \mu_{1}(z) & =\mathfrak{F}_{1}\left(z, \mu_{2}(z), D^{\varsigma} \mu_{2}(z)\right), \\
D^{\theta_{2}} \mu_{1}(z) & =\mathfrak{\mho}_{2}\left(z, \mu_{1}(z), D^{\nu} \mu_{1}(z)\right), \quad 0<z<1, \\
\mu_{1}(0) & =\mu_{1}(1)=\mu_{2}(0)=\mu_{2}(1)=0,
\end{aligned}
$$

where $D^{\theta_{1}}, D^{\varsigma}, D^{\theta_{2}}$, and $D^{v}$ denote Riemann-Liouville derivatives, $1<\theta_{1}, \theta_{2} \leq 2, \mathfrak{F}_{1}, \mathfrak{F}_{2}:[0,1] \times \mathscr{R} \times \mathscr{R} \rightarrow$ $\mathscr{R}$ are continuous functions, and $\mu, \nu$ satisfy $\theta_{1}-\varsigma$ and $\theta_{2}-v \geq 1$. Wang et al. [14] investigate existence and uniqueness of positive solutions to a coupled system of 
fractional differential equations with three-point boundary conditions. The corresponding problem is given as follows:

$$
\begin{aligned}
& D^{\theta_{1}} \mu_{1}(z)=\mathfrak{F}_{1}\left(z, \mu_{2}(z)\right), \\
& D^{\theta_{2}} \mu_{2}(z)=\mathfrak{F}_{2}\left(z, \mu_{1}(z)\right), \\
& \quad 0<z<1, \\
& \mu_{1}(0)=0, \\
& \mu_{2}(0)=0, \\
& \mu_{1}(1)=a \mu_{1}(\eta), \\
& \mu_{2}(1)=b \mu_{2}(\eta),
\end{aligned}
$$

where functions $\mathfrak{F}_{1}, \mathfrak{F}_{2}:[0,1] \times[0, \infty) \rightarrow[0, \infty)$ are continuous, $1<\theta_{1}, \theta_{2} \leq 2,0 \leq a, b \leq 1,0<\eta<$ 1 , and $D^{\theta_{1}}, D^{\theta_{2}}$ represent Riemann-Liouville derivatives. Liu et al. [15] studied existence of positive solutions to a coupled system of nonlinear FODEs with integral boundary conditions. The considered problem is given in the following sequel:

$$
\begin{array}{rlr}
D^{\theta_{1}} \mu_{1}(z) & =\mathfrak{F}_{1}\left(z, \mu_{1}(z), \mu_{2}(z)\right), & \\
D^{\theta_{2}} \mu_{2}(z) & =\mathfrak{F}_{2}\left(z, \mu_{1}(z), \mu_{2}(z)\right), & 0<z<1, \\
\mu_{1}(0) & =0, & \\
\mu_{1}(1) & =\int_{0}^{1} \phi(z) \mu_{1}(z) d z, & 0<z<1 \\
\mu_{2}(0) & =0, & \\
\mu_{2}(1) & =\int_{0}^{1} \varphi(z) \mu_{2}(z) d z, & 0<z<1,
\end{array}
$$

where $D^{\theta_{1}}, D^{\theta_{2}}$ are in sense of Riemann-Liouville derivatives, $\mathfrak{F}_{1}, \mathfrak{F}_{2}:(0,1) \times(0,+\infty) \times(0,+\infty) \rightarrow(0,+\infty)$, and $\phi, \varphi \in$ $L^{1}(0,1)$ are nonlocal functions. The existence and uniqueness of solutions of FODEs are an active area of research for the last few decades. For some remarkable work, we refer the reader to $[8,9,13,16-20]$.

Another qualitative aspect which is very important from the numerical and optimization point of view is devoted to stability analysis of FODEs. The stability of fractional differential equations has gained great attention from the researchers very recently. Different kinds of stability include exponential, Mittag-Leffler, and Lyapunov stabilities; see [2123]. One of the most relaxed methods for stability for functional equations was introduced by Ulam [24] and Hyers [25] which is known as Hyers-Ulam stability. The aforesaid stability has been very well investigated for ordinary differential and integral equations as well as functional equations; see [26-29]. But for FODEs, the concerned stability is not properly investigated. Very few papers can be found in literature in which some initial and boundary value problems of FODEs have been considered; see [7, 23, 30-32].
Motivated by the aforementioned contributions of researchers, we discuss the existence and uniqueness of solutions for coupled system of nonlinear FODEs with boundary conditions involving fractional integral and derivative. Further, we also investigate the Hyers-Ulam stability for the proposed problem designed by

$$
\begin{aligned}
D^{\theta_{1}} \mu_{1}(z) & =\mathfrak{F}_{1}\left(z, \mu_{1}(z), \mu_{2}(z)\right) \quad 0<z<1, \\
D^{\theta_{2}} \mu_{2}(z) & =\mathfrak{F}_{2}\left(z, \mu_{1}(z), \mu_{2}(z)\right), \quad 0<z<1, \\
\mu_{1}(0) & =\mu_{1}^{\prime}(0)=\mu_{1}^{\prime \prime}(0)=\cdots=\mu_{1}^{(n-2)}(0)=\mu_{1}(1) \\
& =0, \\
\mu_{2}(0) & =\mu_{2}^{\prime}(0)=\mu_{2}^{\prime \prime}(0)=\cdots=\mu_{2}^{(n-2)}(0)=\mu_{2}(1) \\
& =0,
\end{aligned}
$$

where the derivative $D$ is in sense of Riemann-Liouville, $n-1<\theta_{1}, \theta_{2} \leq n, n \geq 2$, and the functions $\mathfrak{F}_{1}, \mathfrak{F}_{2}$ : $I \times \mathscr{R} \times \mathscr{R} \rightarrow \mathscr{R}$ are continuous. We use Perov's fixed point theorem [33] and Leray-Schauder fixed point theorem to develop some results for existence of at least one solution for our proposed coupled nonlinear FODEs with boundary conditions. Further, we establish some conditions for HyersUlam type stability to the considered problem. The whole analysis is then demonstrated by providing a proper example.

\section{Preliminaries}

Here we provide some results and definitions for our proposed coupled nonlinear FODEs with boundary conditions from literature [1-3].

Definition 1. The fractional integral of order $\theta_{1} \in \mathscr{R}^{+}$of a function $\mathfrak{F}:(0, \infty) \rightarrow \mathscr{R}$ is defined by

$$
I^{\theta_{1}} \mathfrak{F}(z)=\frac{1}{\Gamma\left(\theta_{1}\right)} \int_{0}^{z}(z-\hbar)^{\theta_{1}-1} \mathfrak{F}(\hbar) d \hbar,
$$

provided that the integral on right is converging.

Definition 2. The Riemann-Liouville fractional order derivative of a function $\mathfrak{F}:(0, \infty) \rightarrow \mathscr{R}$ is defined by

$$
D^{\theta_{1}} \mathfrak{F}(z)=\frac{1}{\Gamma\left(n-\theta_{1}\right)} \frac{d^{n}}{d t^{n}} \int_{0}^{z}(z-\hbar)^{n-\theta_{1}-1} \mathfrak{F}(\hbar) d \hbar,
$$

where $n=\left[\theta_{1}\right]+1$ and $\left[\theta_{1}\right]$ represents the integer part of $\theta_{1}$, provided that the right side is pointwise defined on $(0, \infty)$.

Lemma 3. The following result holds for fractional derivative and integral:

$$
\begin{aligned}
I^{\theta_{1}} D^{\theta_{1}} \mathfrak{F}(z)= & \mathfrak{F}(z)+c_{1} z^{\theta_{1}-1}+c_{2} z^{\theta_{1}-2}+c_{3} z^{\theta_{1}-3}+\cdots \\
& +c_{n} z^{\theta_{1}-n}
\end{aligned}
$$

for arbitrary $c_{i} \in \mathscr{R}, i=1,2, \ldots, n$. 
Lemma 4 (see [20]). Let $\chi$ be a Banach space with $\mathbb{P} \subseteq \chi$ closed and convex. Let $\partial$ be a relatively open subset of $\mathbb{P}$ with $0 \in \partial$ and $\mathscr{T}: \bar{\partial} \rightarrow$ be a continuous and compact (completely continuous) mapping. Then either

(1) the mapping $\mathscr{T}$ has a fixed point in $\bar{ð}$ or

(2) there exist $\mu_{1} \in \partial \partial$ and $\lambda \in(0,1)$ with $\lambda=\lambda \mathscr{T} \mu_{1}$.

Definition 5. For a nonempty set $\mathbb{Z}$, a mapping $d: \mathbb{Z} \times$ $\mathbb{Z} \rightarrow \mathscr{R}^{n}$ is called a generalized metric on $\mathbb{Z}$ if the following conditions hold:

$$
\begin{aligned}
& \left(M_{1}\right) d\left(\mu_{1}, \mu_{2}\right)=0_{R^{n}} \Leftrightarrow \mu_{1}=\mu_{2}, \forall \mu_{1}, \mu_{2} \in X . \\
& \left(M_{2}\right) d\left(\mu_{1}, \mu_{2}\right)=d\left(\mu_{2}, \mu_{1}\right), \forall \mu_{1}, \mu_{2} \in X, \text { (symmetric prop- } \\
& \text { erty). } \\
& \left(M_{3}\right) d(x, y) \leq d\left(x, \mu_{2}\right)+d\left(\mu_{2}, \mu_{1}\right)+d\left(\mu_{1}, y\right), \forall x, y, \mu_{1}, \\
& \mu_{2} \in X \text { (tetrahedral inequality). }
\end{aligned}
$$

Note. The properties such as convergent sequence, cauchy sequence, and open/closed subset are the same for generalized metric spaces as held for the usual metric spaces.

Definition 6. For an $n \times n$ matrix $A$, the spectral radius is defined by $\rho(A)=\max \left\{\left|\eta_{i}\right|, i=1,2, \ldots, n\right\}$, where $\eta_{i}, \quad(i=$ $1,2, \ldots, n)$ are the eigenvalues of matrix $A$.

Lemma 7 (see [33]). Let $(Z, d)$ be a complete generalized metric space and let $\mathscr{T}: Z \rightarrow Z$ be an operator such that there exists a matrix $A \in M$ with $d\left(\mathscr{T} \mu_{1}, \mathscr{T} \mu_{2}\right) \leq$ $\operatorname{Ad}\left(\mu_{1}, \mu_{2}\right)$, for all $\mu_{1}, \mu_{2} \in Z$. If $\rho(A)<1$, then $\mathscr{T}$ has a fixed point $\mu^{*} \in Z$; further for any $\mu_{0}$ the iterative sequence $\mu_{n+1}=\mathscr{T} \mu_{n}$ converges to $\mu_{0}$.

Definition 8. Consider a Banach space $\mathscr{W}_{1} \times \mathscr{W}_{2}$ such that $\mathscr{T}_{1}, \mathscr{T}_{2}: \mathscr{W}_{1} \times \mathscr{W}_{2} \rightarrow \mathscr{W}_{1} \times \mathscr{W}_{2}$ be two operators. Then the operatorial system provided by

$$
\begin{aligned}
& \mu_{1}(z)=\mathscr{T}_{1}\left(\mu_{1}, \mu_{2}\right)(z), \\
& \mu_{2}(z)=\mathscr{T}_{2}\left(\mu_{1}, \mu_{2}\right)(z)
\end{aligned}
$$

is called Hyers-Ulam stable if we can find $\Lambda_{i}(i=1,2,3,4)>$ 0 , such that, for each $\varepsilon_{i}(i=1,2)>0$ and for each solution $\left(\mu_{1}^{*}, \mu_{2}^{*}\right) \in E$ of the inequalities given by

$$
\begin{aligned}
& \left\|\mu_{1}^{*}-\mathscr{T}_{1}\left(\mu_{1}^{*}, \mu_{2}^{*}\right)\right\|_{\infty} \leq \varepsilon_{1}, \\
& \left\|\mu_{2}^{*}-\mathscr{T}_{2}\left(\mu_{1}^{*}, \mu_{2}^{*}\right)\right\|_{\infty} \leq \varepsilon_{2},
\end{aligned}
$$

there exists a solution $\left(\overline{\mu_{1}}, \overline{\mu_{2}}\right) \in E$ of system (8) which satisfies

$$
\begin{aligned}
& \left\|\mu_{1}^{*}-\overline{\mu_{1}}\right\|_{\infty} \leq \Lambda_{1} \varepsilon_{1}+\Lambda_{2} \varepsilon_{2}, \\
& \left\|\mu_{2}^{*}-\overline{\mu_{2}}\right\|_{\infty} \leq \Lambda_{3} \varepsilon_{1}+\Lambda_{4} \varepsilon_{2} .
\end{aligned}
$$

Theorem 9 (see [26]). Considering a Banach space $\mathscr{W}_{1} \times \mathscr{W}_{2}$ with $\mathscr{T}_{1}, \mathscr{T}_{2}: \mathscr{W}_{1} \times \mathscr{W}_{2} \rightarrow \mathscr{W}_{1} \times \mathscr{W}_{2}$ being two operators such that

$$
\begin{aligned}
& \left\|\mathscr{T}_{1}\left(\mu_{1}, \mu_{2}\right)-\mathscr{T}_{1}\left(\mu_{1}^{*}, \mu_{2}^{*}\right)\right\|_{\infty} \\
& \leq \Lambda_{1}\left\|\mu_{1}-\mu_{1}^{*}\right\|_{\infty}+\Lambda_{2}\left\|\mu_{2}-\mu_{2}^{*}\right\|_{\infty}, \\
& \left\|\mathscr{T}_{2}\left(\mu_{1}, \mu_{2}\right)-\mathscr{T}_{2}\left(\mu_{1}^{*}, \mu_{2}^{*}\right)\right\|_{\infty} \\
& \leq \Lambda_{3}\left\|\mu_{1}-\mu_{1}^{*}\right\|_{\infty}+\Lambda_{4}\left\|\mu_{2}-\mu_{2}^{*}\right\|_{\infty}, \\
& \forall\left(\mu_{1}, \mu_{2}\right),\left(\mu_{1}^{*}, \mu_{2}^{*}\right) \in \mathscr{W}_{1} \times \mathscr{W}_{2},
\end{aligned}
$$

and if the matrix $\left(\begin{array}{ll}\Lambda_{1} & \Lambda_{2} \\ \Lambda_{3} & \Lambda_{4}\end{array}\right)$ converges to zero, then the operatorial system (8) is Hyers-Ulam stable.

Lemma 10. An equivalent Fredholm integral representation of the system of boundary value problems (4) is given by

$$
\begin{aligned}
& \mu_{1}(z)=\int_{0}^{1} G_{\theta_{1}}(z, \hbar) \mathfrak{\mho}_{1}\left(\hbar, \mu_{1}(\hbar), \mu_{2}(\hbar)\right) d \hbar, \\
& \mu_{2}(z)=\int_{0}^{1} G_{\theta_{2}}(z, \hbar) \mathfrak{\mho}_{2}\left(\hbar, \mu_{1}(\hbar), \mu_{2}(\hbar)\right) d \hbar,
\end{aligned}
$$

where $G_{\theta_{1}}, G_{\theta_{2}}$ are Green's functions given by

$$
\begin{aligned}
& G_{\theta_{1}}(z, \hbar) \\
& = \begin{cases}\frac{[z(1-\hbar)]^{\theta_{1}-1}}{\Gamma\left(\theta_{1}\right)}, & 0 \leq z \leq \hbar \leq 1, \\
\frac{[z(1-\hbar)]^{\theta_{1}-1}}{\Gamma\left(\theta_{1}\right)}-\frac{(z-\hbar)^{\theta_{1}-1}}{\Gamma\left(\theta_{1}\right)}, & 0 \leq \hbar \leq z \leq 1,\end{cases} \\
& G_{\theta_{2}}(z, \hbar) \\
& = \begin{cases}\frac{[z(1-\hbar)]^{\theta_{2}-1}}{\Gamma\left(\theta_{2}\right)}, & 0 \leq z \leq \hbar \leq 1, \\
\frac{[z(1-\hbar)]^{\theta_{2}-1}}{\Gamma\left(\theta_{2}\right)}-\frac{(z-\hbar)^{\theta_{2}-1}}{\Gamma\left(\theta_{2}\right)}, & 0 \leq \hbar \leq z \leq 1 .\end{cases}
\end{aligned}
$$

Proof. Applying the operator $I^{\theta_{1}}$ on the first equation of (4) and using Lemma 3 , we have

$$
\begin{array}{r}
\mu_{1}(z)=-I^{\theta_{1}} \mathfrak{F}_{1}\left(z, \mu_{1}(z), \mu_{2}(z)\right)+c_{1} z^{\theta_{1}-1}+c_{2} z^{\theta_{1}-2} \\
+c_{3} z^{\theta_{1}-3}+\cdots+c_{n} z^{\theta_{1}-n}, \\
c_{i} \in \mathscr{R}, \text { for } i=1,2, \ldots, n .
\end{array}
$$

The boundary conditions $\mu_{1}(0)=\mu_{1}^{\prime}(0)=\mu_{1}^{\prime \prime}(0)=\cdots=$ $\mu_{1}^{(n-2)}(0)=\mu_{1}(1)=0$ and they yield $c_{n}=\cdots=c_{2}=0$ due to 
singularity and $c_{1}=I^{\theta_{1}} \mathfrak{F}_{1}\left(1, \mu_{1}(1), \mu_{2}(1)\right)$. Hence, (14) takes the form

$$
\begin{aligned}
\mu_{1}(z) & \\
= & \frac{z^{\theta_{1}-1}}{\Gamma\left(\theta_{1}\right)} \int_{0}^{1}(1-\hbar)^{\theta_{1}-1} \mathfrak{F}_{1}\left(\hbar, \mu_{1}(\hbar), \mu_{2}(\hbar)\right) d \hbar \\
& \quad-\frac{1}{\Gamma\left(\theta_{1}\right)} \int_{0}^{t}(z-\hbar)^{\theta_{1}-1} \mathfrak{F}_{1}\left(\hbar, \mu_{1}(\hbar), \mu_{2}(\hbar)\right) d \hbar \\
= & \int_{0}^{1} G_{\theta_{1}}(z, \hbar) \mathfrak{F}_{1}\left(\hbar, \mu_{1}(\hbar), \mu_{2}(\hbar)\right) d \hbar .
\end{aligned}
$$

Similarly, by the same process with the second equation of the system, we obtain the second part of (12).

Lemma 11 (see [18]). Green's function $G=\left(G_{\theta_{1}}, G_{\theta_{2}}\right)$ of system (12) has the following properties:

$\left(C_{1}\right) G(z, \hbar)$ is continuous function on the unit square for all $(z, \hbar) \in[0,1] \times[0,1]$.

$\left(C_{2}\right) G(z, \hbar) \geq 0$ for all $z, \hbar \in[0,1]$ and $G(z, \hbar)>0$ for all $z, \hbar \in(0,1)$.

$\left(C_{3}\right) \max _{0 \leq z \leq 1} G(z, \hbar)=G(1, \hbar), \quad \forall \hbar \in[0,1]$.

$\left(C_{4}\right)$ There exists a constant $\gamma \in(0,1)$ such that

$$
\min _{z \in[\theta, 1-\theta]} G(z, \hbar) \geq \gamma(\hbar) G(1, \hbar) \quad \text { for } \theta \in(0,1), \hbar \in[0,1] \text { where } \gamma=\min \left\{\gamma_{\theta_{1}}=\left(\frac{z}{\hbar}\right)^{\theta_{1}-1}, \gamma_{\theta_{2}}=\left(\frac{z}{\hbar}\right)^{\theta_{2}-1}\right\}
$$

\section{Existence and Hyers-Ulam Stability}

Define $\mathbb{U}=\left\{\mu_{1}(z) \quad \mid \mu_{1}(z) \in C[0,1]\right\}$ endowed with the Chebyshev norm $\left\|\mu_{1}\right\|_{\infty}=\max _{t \in[0,1]}\left|\mu_{1}(z)\right|$. Further, define the norms $\left\|\left(\mu_{1}, \mu_{2}\right)\right\|_{\mathscr{W}_{1} \times \mathscr{W}_{2}}=\left\|\mu_{1}\right\|_{\infty}+\left\|\mu_{2}\right\|_{\infty}$ and $\left|\left(\mu_{1}, \mu_{2}\right)\right|_{\mathscr{W}_{1} \times \mathscr{W}_{2}}=\max \left\{\left\|\mu_{1}\right\|_{\infty},\left\|\mu_{2}\right\|_{\infty}\right\}$. Then, the product spaces $\left(\mathscr{W}_{1} \times \mathscr{W}_{2},\|\cdot\|_{\mathscr{W}_{1} \times \mathscr{W}_{2}}\right),\left(\mathscr{W}_{1} \times \mathscr{W}_{2},|\cdot|_{\mathscr{W}_{1} \times \mathscr{W}_{2}}\right)$ are Banach spaces. Define the cones $\mathbb{P}, \mathscr{K} \subset \mathscr{W}_{1} \times \mathscr{W}_{2}$ by $\mathbb{P}=$ $\left\{\left(\mu_{1}, \mu_{2}\right) \in \mathscr{W}_{1} \times \mathscr{W}_{2}: \mu_{1}(z), \mu_{2}(z) \geq 0, \forall z \in[0,1]\right\}$ and $\mathscr{K}=$ $\left\{\left(\mu_{1}, \mu_{2}\right) \in \mathbb{P}: \min _{z \in J}\left[\mu_{1}(z)+\mu_{2}(z)\right] \geq \gamma\left\|\left(\mu_{1}, \mu_{2}\right)\right\|_{\mathscr{W}_{1} \times \mathscr{W}_{2}}\right\}$ where $J=[\theta, 1-\theta], \theta \in(0,1)$.

Lemma 12. Assume that $\mathfrak{\mho}_{1}, \mathfrak{\mho}_{2}:[0,1] \times \mathscr{R} \times \mathscr{R} \rightarrow \mathscr{R}$ are continuous. Then $\left(\mu_{1}, \mu_{2}\right) \in \mathscr{W}_{1} \times \mathscr{W}_{2}$ is a solution of (12), if and only if $\left(\mu_{1}, \mu_{2}\right) \in \mathscr{W}_{1} \times \mathscr{W}_{2}$ is a solution of system of Fredholm integral equations (4).

Proof. The proof of Lemma 12 is similar to proof of Lemma 3.1 in [18].

Define $\mathscr{T}: \mathscr{W}_{1} \times \mathscr{W}_{2} \rightarrow \mathscr{W}_{1} \times \mathscr{W}_{2}$ by

$$
\begin{aligned}
\mathscr{T} & \left(\mu_{1}, \mu_{2}\right)(z) \\
& =\left(\int_{0}^{1} G_{\theta_{1}}(z, \hbar) \mathfrak{F}_{1}\left(\hbar, \mu_{1}(\hbar), \mu_{2}(\hbar)\right) d \hbar,\right. \\
& \left.\int_{0}^{1} G_{\theta_{2}}(z, \hbar) \mathfrak{F}_{2}\left(\hbar, \mu_{1}(\hbar), \mu_{2}(\hbar)\right) d \hbar\right) \\
& =\left(\mathscr{T}_{1}\left(\mu_{1}, \mu_{2}\right)(z), \mathscr{T}_{2}\left(\mu_{1}, \mu_{2}\right)(z)\right) .
\end{aligned}
$$

By Lemma 12 the problem of existence of solutions of the integral equations (12) coincides with the problem of existence of fixed points of $\mathscr{T}$.

Lemma 13. Assume that $\mathfrak{F}_{1}, \mathfrak{F}_{2}:[0,1] \times[0, \infty) \times[0, \infty) \rightarrow$ $[0, \infty)$ are continuous. Then $\mathscr{T}(\mathbb{P}) \subset \mathbb{P}$ and $\mathscr{T}(\mathscr{K}) \subset \mathscr{K}$, where $\mathscr{T}$ is defined by (17).
Proof. The relation $\mathscr{T}(\mathbb{P}) \subset \mathbb{P}$ easily follows from the properties $\left(C_{1}\right)$ and $\left(C_{2}\right)$ of Lemma 11 and all we need to show is that $\mathscr{T}(\mathscr{K}) \subset \mathscr{K}$ holds. For $\left(\mu_{1}, \mu_{2}\right) \in \mathscr{K}$, we have $\mathscr{T}\left(\mu_{1}, \mu_{2}\right) \in \mathbb{P}$ and in view of property $\left(C_{4}\right)$ of Lemma 11 , for all $z \in J$, we obtain

$$
\begin{aligned}
& \mathscr{T}_{1}\left(\mu_{1}(z), \mu_{2}(z)\right) \\
& =\int_{0}^{1} \mathscr{G}_{\theta_{1}}(z, \hbar) \mathfrak{F}_{1}\left(\hbar, \kappa_{1}(\hbar), \mu_{2}(\hbar)\right) d \hbar \\
& \quad \geq \gamma_{\theta_{1}} \int_{0}^{1} G_{\theta_{1}}(1, \hbar) \mathfrak{\mho}_{1}\left(\hbar, \mu_{1}(\hbar), \mu_{2}(\hbar)\right) d \hbar .
\end{aligned}
$$

Hence, it follows that

$$
\min _{z \in J} \mathscr{T}_{1}\left(\mu_{1}(z), \mu_{2}(z)\right) \geq \gamma_{\theta_{1}}\left\|\mathscr{T}_{1}\left(\mu_{1}, \mu_{2}\right)\right\|_{\infty},
$$

$\forall z \in J$.

Similarly, we obtain

$$
\min _{z \in J} \mathscr{T}_{2}\left(\mu_{1}(z), \mu_{2}(z)\right) \geq \gamma_{\theta_{2}}\left\|\mathscr{T}_{2}\left(\mu_{1}, \mu_{2}\right)\right\|_{\infty},
$$

$\forall z \in J$.

It follows that

$$
\begin{aligned}
& \min _{z \in J} {\left[\mathscr{T}_{1}\left(\mu_{1}(z), \mu_{2}(z)\right)+\mathscr{T}_{2}\left(\mu_{1}(z), \mu_{2}(z)\right)\right] } \\
& \geq \gamma\left\|\mathscr{T}_{1}\left(\mu_{1}, \mu_{2}\right), \mathscr{T}_{2}\left(\mu_{1}, \mu_{2}\right)\right\|_{\mathscr{W}_{1} \times \mathscr{W}_{2}}, \quad \forall z \in J,
\end{aligned}
$$

which implies that $\mathscr{T}\left(\mu_{1}, \mu_{2}\right) \in \mathscr{K}$.

Lemma 14. Assume that $\mathfrak{F}_{1}, \mathfrak{F}_{2}:[0,1] \times \mathscr{R} \times \mathscr{R} \rightarrow \mathscr{R}$ are continuous; then $\mathscr{T}: \mathbb{P} \rightarrow \mathbb{P}$ is completely continuous.

Proof. We omit the proof, because it is similar to the proof of Lemma 3.2 in [18].

Lemma 15. Assume that $\mathfrak{F}_{1}$ and $\mathfrak{F}_{2}$ are continuous on $[0,1] \times$ $\mathscr{R} \times \mathscr{R} \rightarrow \mathscr{R}$ and there exist $\phi_{i}, \psi_{i}(i=1,2):(0,1) \rightarrow[0, \infty)$ such that the following hold: 
$\left(C_{1}^{*}\right)\left|\mathfrak{F}_{1}\left(z, \mu_{1}, \mu_{2}\right)-\mathfrak{F}_{1}\left(z, \overline{\mu_{1}}, \overline{\mu_{2}}\right)\right| \leq \phi_{1}(z)\left|\mu_{1}-\overline{\mu_{1}}\right|+$ $\psi_{1}(z)\left|\mu_{2}-\overline{\mu_{2}}\right|, z \in(0,1)$, for $\mu_{1}, \mu_{2}, \overline{\mu_{1}}, \overline{\mu_{2}} \geq 0$;

$\left(C_{2}^{*}\right)\left|\mathfrak{F}_{2}\left(z, \mu_{1}, \mu_{2}\right)-\mathfrak{\mho}_{2}\left(z, \overline{\mu_{1}}, \overline{\mu_{2}}\right)\right| \leq \phi_{2}(z)\left|\mu_{1}-\overline{\mu_{1}}\right|+$ $\psi_{2}(z)\left|\mu_{2}-\overline{\mu_{2}}\right|$, for $z \in(0,1), \mu_{1}, \mu_{2}, \overline{\mu_{1}}, \overline{\mu_{2}} \geq 0$.

$\left(C_{3}^{*}\right) \rho(A)<1$, where the matrix $A \in M_{2,2}\left(\mathscr{R}_{+}\right)$is defined by

A

$$
=\left[\begin{array}{l}
\int_{0}^{1} G_{\theta_{1}}(1, \hbar) \phi_{1}(\hbar) d \hbar \int_{0}^{1} G_{\theta_{1}}(1, \hbar) \psi_{1}(\hbar) d \hbar \\
\int_{0}^{1} G_{\theta_{2}}(1, \hbar) \phi_{2}(\hbar) d \hbar \int_{0}^{1} G_{\theta_{2}}(1, \hbar) \psi_{2}(\hbar) d \hbar
\end{array}\right] .
$$

Then system (12) has a unique positive solution $\left(\mu_{1}, \mu_{2}\right) \in \mathbb{P}$.

Proof. In view of Definition 5, we define the generalized metric $d$ by

$$
\begin{aligned}
d\left(\left(\mu_{1}, \mu_{2}\right),\left(\overline{\mu_{1}}, \overline{\mu_{2}}\right)\right) & =\left(\begin{array}{l}
\left\|\mu_{1}-\overline{\mu_{1}}\right\|_{\infty} \\
\left\|\mu_{2}-\overline{\mu_{2}}\right\|_{\infty}
\end{array}\right), \\
& \forall\left(\mu_{1}, \mu_{2}\right),\left(\overline{\mu_{1}}, \overline{\mu_{2}}\right) \in \mathscr{W}_{1} \times \mathscr{W}_{2} .
\end{aligned}
$$

Obviously $\left(\mathscr{W}_{1} \times \mathscr{W}_{2}, d\right)$ is a generalized complete metric space. For any $\left(\mu_{1}, \mu_{2}\right),\left(\overline{\mu_{1}}, \overline{\mu_{2}}\right) \in \mathscr{W}_{1} \times \mathscr{W}_{2}$ using properties $\left(C_{3}\right)$ and $\left(C_{3}^{*}\right)$, we obtain

$$
\begin{aligned}
& \left|\mathscr{T}_{1}\left(\mu_{1}, \mu_{2}\right)(z)-\mathscr{T}_{1}\left(\overline{\mu_{1}}, \overline{\mu_{2}}\right)(z)\right| \leq \max _{z \in[0,1]} \int_{0}^{1}\left|G_{\theta_{1}}(z, \hbar)\right| \\
& \cdot\left[\left|\mathfrak{F}_{1}\left(\hbar, \mu_{1}(\hbar), \mu_{2}(\hbar)\right)-\mathfrak{F}_{1}\left(\hbar, \overline{\mu_{1}}(\hbar), \overline{\mu_{2}}(\hbar)\right)\right|\right] d \hbar \\
& \leq \int_{0}^{1} G_{\theta_{1}}(1, \hbar)\left[\phi_{1}(\hbar)\left\|\mu_{1}-\overline{\mu_{1}}\right\|_{\infty}+\psi_{1}(\hbar)\right. \\
& \left.\cdot\left\|\mu_{2}-\overline{\mu_{2}}\right\|_{\infty}\right] d \hbar,
\end{aligned}
$$

which implies that

$$
\begin{aligned}
& \left|\mathscr{T}_{1}\left(\mu_{1}, \mu_{2}\right)-\mathscr{T}_{1}\left(\overline{\mu_{1}}, \overline{\mu_{2}}\right)\right| \\
& \leq\left(\int_{0}^{1} G_{1}(1, \hbar) \phi_{1}(\hbar) d \hbar\right)\left\|\mu_{1}-\overline{\mu_{1}}\right\|_{\infty} \\
& \quad+\left(\int_{0}^{1} G_{1}(1, \hbar) \psi_{1}(\hbar) d \hbar\right)\left\|\mu_{2}-\overline{\mu_{2}}\right\|_{\infty} .
\end{aligned}
$$

Similarly, we obtain

$$
\begin{aligned}
& \left|\mathscr{T}_{2}\left(\mu_{1}, \mu_{2}\right)-\mathscr{T}_{2}\left(\overline{\mu_{1}}, \overline{\mu_{2}}\right)\right| \\
& \leq\left(\int_{0}^{1} G_{2}(1, \hbar) \phi_{2}(\hbar) d \hbar\right)\left\|\mu_{1}-\overline{\mu_{1}}\right\|_{\infty} \\
& \quad+\left(\int_{0}^{1} G_{2}(1, \hbar) \psi_{2}(\hbar) d \hbar\right)\left\|\mu_{2}-\overline{\mu_{2}}\right\|_{\infty} .
\end{aligned}
$$

Hence, it follows that

$$
\begin{array}{r}
\left|\mathscr{T}\left(\mu_{1}, \mu_{2}\right)-\mathscr{T}\left(\overline{\mu_{1}}, \overline{\mu_{2}}\right)\right| \leq \operatorname{Ad}\left(\left(\mu_{1}, \mu_{2}\right),\left(\overline{\mu_{1}}, \overline{\mu_{2}}\right)\right), \\
\forall\left(\mu_{1}, \mu_{2}\right),\left(\overline{\mu_{1}}, \overline{\mu_{2}}\right) \in \mathscr{W}_{1} \times \mathscr{W}_{2},
\end{array}
$$

where

A

$$
=\left[\begin{array}{l}
\int_{0}^{1} G_{\theta_{1}}(1, \hbar) \phi_{1}(\hbar) d \hbar \int_{0}^{1} G_{\theta_{1}}(1, \hbar) \psi_{1}(\hbar) d \hbar \\
\int_{0}^{1} G_{\theta_{2}}(1, \hbar) \phi_{2}(\hbar) d \hbar \int_{0}^{1} G_{\theta_{2}}(1, \hbar) \psi_{2}(\hbar) d \hbar
\end{array}\right] .
$$

By $\left(C_{3}^{*}\right), \rho(A)<1$. Hence by Lemma 7 , system (12) has a unique positive solution.

Lemma 16. Let $\mathfrak{F}_{1}$ and $\mathfrak{F}_{2}$ be continuous on $[0,1] \times \mathscr{R} \times \mathscr{R} \rightarrow$ $\mathscr{R}$ and there exist $a_{i}, b_{i}, c_{i}(i=1,2):(0,1) \rightarrow[0, \infty)$ satisfying

$\left(C_{4}^{*}\right)\left|\mathfrak{\mho}_{1}\left(z, \mu_{1}(z), \mu_{2}(z)\right)\right| \leq a_{1}(z)+b_{1}(z)\left|\mu_{1}(z)\right|+c_{1}(z)\left|\mu_{2}(z)\right|$, $z \in(0,1), \mu_{1}, \mu_{2} \geq 0$

$\left(C_{5}^{*}\right)\left|\mathfrak{\mho}_{2}\left(z, \mu_{1}(z), \mu_{2}(z)\right)\right| \leq a_{2}(z)+b_{2}(z)\left(\left|\mu_{1}(z)\right|+c_{2}\left|\mu_{2}(z)\right|\right.$, $z \in(0,1), \mu_{1}, \mu_{2} \geq 0$

$\left(C_{6}^{*}\right) \mathbb{A}_{1}=\int_{0}^{1} G_{\theta_{1}}(1, \hbar) a_{1}(\hbar) d \hbar<\infty, \mathbb{B}_{1}=\int_{0}^{1} G_{\theta_{1}}(1$, $\hbar)\left[b_{1}(\hbar)+c(\hbar)\right] d \hbar<1$

$\left(C_{7}^{*}\right) \mathbb{A}_{2}=\int_{0}^{1} G_{\theta_{2}}(1, \hbar) a_{2}(\hbar) d \hbar<\infty, \mathbb{B}_{2}=\int_{0}^{1} G_{\theta_{2}}(1$, $\hbar)\left[b_{2}(\hbar)+c_{2}(\hbar)\right] d \hbar<1$.

Then system (12) has at least one positive solution $\left(\mu_{1}, \mu_{2}\right)$ in

$$
\begin{aligned}
\mathscr{Q} & =\left\{\left(\mu_{1}, \mu_{2}\right) \in \mathbb{P}:\left(\mu_{1}, \mu_{2}\right)\right. \\
& \left.<\min \left(\frac{2 \mathbb{A}_{1}}{1-2 \mathbb{B}_{1}}, \frac{2 \mathbb{A}_{2}}{1-2 \mathbb{B}_{2}}\right)\right\} .
\end{aligned}
$$

Proof. Choose $r=\min \left(2 \mathbb{A}_{1} /\left(1-2 \mathbb{B}_{1}\right), 2 \mathbb{A}_{2} /\left(1-2 \mathbb{B}_{2}\right)\right)$ and define $\partial=\left\{\left(\mu_{1}, \mu_{2}\right) \in \mathbb{P}:\left\|\left(\mu_{1}, \mu_{2}\right)\right\|<r\right\}$. By Lemma 14, the operator $\mathscr{T}: \bar{\partial} \rightarrow \mathbb{P}$ is completely continuous. Choose $\lambda \in$ $(0,1)$ and $\left(\mu_{1}, \mu_{2}\right) \in \partial ð$ such that $\left(\mu_{1}, \mu_{2}\right)=\lambda \mathscr{T}\left(\mu_{1}, \mu_{2}\right)$. Then, by properties $\left(C_{1}\right),\left(C_{3}\right)$, and $\left(C_{4}^{*}\right)$, we obtain for all $z \in[0,1]$

$$
\begin{aligned}
& \left\|\mu_{1}(z)\right\|_{\infty} \leq \lambda \max _{z \in[0,1]} \int_{0}^{1} G_{\theta_{1}}(z, \hbar) \\
& \cdot\left|\mathfrak{F}_{1}(\hbar), \mu_{1}(\hbar), \mu_{2}(\hbar) d \hbar\right| \\
& \leq \lambda\left[\int_{0}^{1} \mathscr{G}_{\theta_{1}}(1, \hbar) a_{1}(\hbar)\right. \\
& \left.+\int_{0}^{1} \mathscr{G}_{\theta_{1}}(1, \hbar)\left(b_{1}(\hbar)\left|\mu_{1}(\hbar)\right|+c_{1}(\hbar)\left|\mu_{2}(\hbar)\right|\right)\right] d \hbar \\
& \leq\left(\mathbb{A}_{1}+r \mathbb{B}_{1}\right) \leq \frac{r}{2} .
\end{aligned}
$$

Similarly, we obtain $\left\|\mu_{2}\right\|_{\infty} \leq r / 2$; hence $\left\|\left(\mu_{1}, \mu_{2}\right)\right\|<r$, which shows that $\left(\mu_{1}, \mu_{2}\right) \notin \partial \partial$. Thus, by Schauder fixed point theorem, $\mathscr{T}$ has at least one fixed point in $\bar{\varnothing}$.

\section{Hyers-Ulam Stability of (12)}

In this section, we obtain some appropriate conditions under which the toppled system under our consideration is HyersUlam stable. 
Let the following assumption hold:

$\left(C_{8}^{*}\right)$ There exist constants $\mathfrak{Q}_{i}>0, \mathfrak{\Omega}_{i}>0, i=1,2$, such that

$$
\begin{gathered}
\left|\mathfrak{F}_{1}\left(z, \mu_{1}, \mu_{2}\right)-\mathfrak{F}_{1}\left(z, \bar{\mu}_{1}, \bar{\mu}_{2}\right)\right| \\
\leq \mathfrak{Q}_{1}\left|\mu_{1}-\overline{\mu_{1}}\right|+\mathfrak{Q}_{2}\left|\mu_{2}-\overline{\mu_{2}}\right|, \\
\left|\mathfrak{F}_{2}\left(z, \mu_{1}, \mu_{2}\right)-\mathfrak{F}_{2}\left(z, \bar{\mu}_{1}, \bar{\mu}_{2}\right)\right| \\
\leq \mathfrak{R}_{1}\left|\mu_{1}-\overline{\mu_{1}}\right|+\mathfrak{\Re}_{2}\left|\mu_{2}-\overline{\mu_{2}}\right| .
\end{gathered}
$$

Theorem 17. Assume that hypothesis $\left(C_{8}^{*}\right)$ holds and $\mathscr{T}_{1}, \mathscr{T}_{2}$ : $\mathscr{W}_{1} \times \mathscr{W}_{2} \rightarrow \mathscr{W}_{1} \times \mathscr{W}_{2}$ such that

$$
\begin{aligned}
& \left\|\mathscr{T}_{1}\left(\mu_{1}, \mu_{2}\right)-\mathscr{T}_{1}\left(\mu_{1}^{*}, \mu_{2}^{*}\right)\right\|_{\infty} \\
& \quad \leq \Lambda_{1}\left\|\mu_{1}-\mu_{1}^{*}\right\|_{\infty}+\Lambda_{2}\left\|\mu_{2}-\mu_{2}^{*}\right\|_{\infty}, \\
& \left\|\mathscr{T}_{2}\left(\mu_{1}, \mu_{2}\right)-\mathscr{T}_{2}\left(\mu_{1}^{*}, \mu_{2}^{*}\right)\right\|_{\infty} \\
& \quad \leq \Lambda_{3}\left\|\mu_{1}-\mu_{1}^{*}\right\|_{\infty}+\Lambda_{4}\left\|\mu_{2}-\mu_{2}^{*}\right\|_{\infty},
\end{aligned}
$$

for all solutions $\left(\mu_{1}, \mu_{2}\right),\left(\mu_{1}^{*}, \mu_{2}^{*}\right) \in \mathscr{W}_{1} \times \mathscr{W}_{2}$, with

$$
\mathcal{Q}=\left(\begin{array}{ll}
\Lambda_{1} & \Lambda_{2} \\
\Lambda_{3} & \Lambda_{4}
\end{array}\right),
$$

converges to zero. Then the solution of coupled system (4) is Hyers-Ulam stable.

Proof. Consider

$$
\begin{gathered}
\left|\mathscr{T}_{1}\left(\mu_{1}, \mu_{2}\right)(z)-\mathscr{T}_{1}\left(\overline{\mu_{1}}, \overline{\mu_{2}}\right)(z)\right| \leq \max _{z \in[0,1]} \int_{0}^{1}\left|G_{\theta_{1}}(z, \hbar)\right| \\
\cdot\left[\left|\mathfrak{F}_{1}\left(\hbar, \mu_{1}(\hbar), \mu_{2}(\hbar)\right)-\mathfrak{F}_{1}\left(\hbar, \overline{\mu_{1}}(\hbar), \overline{\mu_{2}}(\hbar)\right)\right|\right] d \hbar \\
\leq \int_{0}^{1} G_{\theta_{1}}(1, \hbar)\left[\mathfrak{Q}_{1}\left\|\mu_{1}-\overline{\mu_{1}}\right\|_{\infty}+\mathfrak{Q}_{2}\left\|\mu_{2}-\overline{\mu_{2}}\right\|_{\infty}\right] d \hbar,
\end{gathered}
$$

which implies that $\left|\mathscr{T}_{1}\left(\mu_{1}, \mu_{2}\right)-\mathscr{T}_{1}\left(\overline{\mu_{1}}, \overline{\mu_{2}}\right)\right| \leq \Lambda_{1}\left\|\mu_{1}-\overline{\mu_{1}}\right\|_{\infty}+$ $\Lambda_{2}\left\|\mu_{2}-\overline{\mu_{2}}\right\|_{\infty}$.

According to the previous sentence, $\Lambda_{1}=\mathfrak{\Omega}_{1} / \Gamma\left(\theta_{1}+\right.$ 1), $\Lambda_{2}=\mathfrak{Q}_{2} / \Gamma\left(\theta_{1}+1\right)$. Similarly, we can also get

$$
\begin{aligned}
& \left\|\mathscr{T}_{2}\left(\mu_{1}, \mu_{2}\right)-\mathscr{T}_{2}\left(\mu_{1}^{*}, \kappa_{2}^{*}\right)\right\|_{\infty} \\
& \quad \leq \Lambda_{3}\left\|\mu_{1}-\overline{\mu_{1}}\right\|_{\infty}+\Lambda_{4}\left\|\mu_{2}-\overline{\mu_{2}}\right\|_{\infty},
\end{aligned}
$$

where $\Lambda_{3}=\mathfrak{\Omega}_{1} / \Gamma\left(\theta_{2}+1\right), \Lambda_{4}=\mathfrak{\Omega}_{2} / \Gamma\left(\theta_{2}+1\right)$. Therefore from (34) and (35), we have the following system of inequalities:

$$
\begin{aligned}
& \left\|\mathscr{T}_{1}\left(\mu_{1}, \mu_{2}\right)-\mathscr{T}_{1}\left(\mu_{1}^{*}, \mu_{2}^{*}\right)\right\|_{\infty} \\
& \quad \leq \Lambda_{1}\left\|\mu_{1}-\mu_{1}^{*}\right\|_{\infty}+\Lambda_{2}\left\|\mu_{2}-\mu_{2}^{*}\right\|_{\infty}, \\
& \left\|\mathscr{T}_{2}\left(\mu_{1}, \mu_{2}\right)-\mathscr{T}_{2}\left(\mu_{1}^{*}, \mu_{2}^{*}\right)\right\|_{\infty} \\
& \quad \leq \Lambda_{3}\left\|\mu_{1}-\mu_{1}^{*}\right\|_{\infty}+\Lambda_{4}\left\|\mu_{2}-\mu_{2}^{*}\right\|_{\infty},
\end{aligned}
$$

where $\mathscr{M}=\left(\begin{array}{ll}\Lambda_{1} & \Lambda_{2} \\ \Lambda_{3} & \Lambda_{4}\end{array}\right)$ which converges to zero. Thus, in view of Theorem 9, the solution of coupled system (4) is Hyers-Ulam stable.

\section{Examples}

Example 1. Consider the following coupled nonlinear FODEs of boundary conditions:

$$
\begin{aligned}
& D^{5 / 2} \mu_{1}(z)+\Gamma\left(\frac{5}{2}\right)\left[\frac{z \mu_{1}(z)}{16}+\frac{z^{2} \mu_{2}(z)}{32}\right]=0, \\
& z \in[0,1], \\
& D^{5 / 2} \mu_{2}(z) \\
& \quad+\Gamma\left(\frac{5}{2}\right)\left[\frac{9 z^{2}\left|\cos \left(\mu_{1}(z)\right)\right|}{16 \sqrt{\pi}}+\frac{9 z\left|\cos \left(\mu_{2}(z)\right)\right|}{32 \sqrt{\pi}}\right] \\
& \quad=0, \quad z \in[0,1], \\
& \mu_{1}(0)=\mu_{1}^{\prime}(0)=\mu_{1}(1)=0, \\
& \mu_{2}(0)=\mu_{2}^{\prime}(0)=\mu_{2}(1)=0 .
\end{aligned}
$$

Here $n=3$ and $\phi_{1}(z)=\Gamma(5 / 2)(z / 16), \psi_{1}(z)=\Gamma(5 / 2)\left(z^{2} /\right.$ $32), \phi_{2}(z)=\Gamma(5 / 2)\left(9 z^{2} / 16 \sqrt{\pi}\right), \psi_{2}(z)=\Gamma(5 / 2)(9 z / 32 \sqrt{\pi})$. Moreover

A

$$
\begin{aligned}
& =\left[\begin{array}{ll}
\int_{0}^{1} G_{\theta_{1}}(1, \hbar) \phi_{1}(\hbar) d \hbar & \int_{0}^{1} G_{\theta_{1}}(1, \hbar) \psi_{1}(\hbar) d \hbar \\
\int_{0}^{1} G_{\theta_{2}}(1, \hbar) \phi_{2}(\hbar) d \hbar \int_{0}^{1} G_{\theta_{2}}(1, \hbar) \psi_{2}(\hbar) d \hbar
\end{array}\right] \\
& =\left[\begin{array}{ll}
0.0460 & 0.0007 \\
0.0068 & 0.0058
\end{array}\right] .
\end{aligned}
$$

Here, $\rho(A)=4.61 \times 10^{-2}<1$; hence by Lemma 15 the BVP (37) has a unique solution. For $\mathfrak{F}_{1}$ and $\mathfrak{F}_{2}$, we have $a_{1}(z)=0$, $b_{1}(z)=\Gamma(5 / 2)(z / 16), c_{1}(z)=\Gamma(5 / 2)\left(z^{2} / 32\right), a_{2}(z)=0$, $b_{2}(z)=\Gamma(5 / 2)\left(9 z^{2} / 16 \sqrt{\pi}\right), c_{2}(z)=\Gamma(5 / 2)(9 z / 32 \sqrt{\pi})$ and, by simple calculation, we obtain

$$
\begin{aligned}
& \mathbb{A}_{1}=\int_{0}^{1} G_{\theta_{1}}(z, \hbar) a_{1}(\hbar) d \hbar<\infty, \\
& \mathbb{A}_{2}=\int_{0}^{1} G_{\theta_{2}}(z, \hbar) a_{2}(\hbar) d \hbar<\infty, \\
& \mathbb{B}_{1}=\int_{0}^{1} G_{\theta_{1}}(z, \hbar)\left[b_{1}(\hbar)+c_{1}(\hbar)\right] d \hbar<1, \\
& \mathbb{B}_{2}=\int_{0}^{1} G_{\theta_{2}}(z, \hbar)\left[b_{2}(\hbar)+c_{2}(\hbar)\right] d \hbar<1 .
\end{aligned}
$$

Hence, by using Lemma 16, BVP (37) has at least one positive solution. Further, it is easy to compute the matrix $\mathbb{Q}$ which converges to zero and so the solution is Hyers-Ulam stable by using Theorem 17.

\section{Conclusion}

In this paper, we investigate existence and uniqueness of solutions for the nonlinear FODEs with boundary conditions 
and also investigate Hyers-Ulam stability for the mentioned problem. We use Perov's fixed point theorem [33] and LeraySchauder fixed point theorem to develop some results for existence of at least one solution for our proposed coupled nonlinear FODEs with boundary conditions. Further, we establish some conditions for Hyers-Ulam type stability to the considered problem. The whole paper is very easy because of relaxed methods and conditions.

\section{Conflicts of Interest}

The authors declare that no conflicts of interest exist regarding this manuscript.

\section{Authors' Contributions}

All authors equally contributed to this paper and approved the final version.

\section{Acknowledgments}

This work has been supported by the National Natural Science Foundation of China (11571378).

\section{References}

[1] I. Podlubny, Fractional Differential Equations, Academic Press, New York, NY, USA, 1999.

[2] R. Hilfer, Applications of Fractional Calculus in Physics, World Scientific, Singapore, 2000.

[3] K. S. Miller and B. Ross, An Introduction to the Fractional Calculus and Fractional Differential Equations, A Wiley-Interscience Publication, John Wiley \& Sons, New York, NY, USA, 1993.

[4] K. B. Oldham, "Fractional differential equations in electrochemistry," Advances in Engineering Software, vol. 41, no. 1, pp. 9-12, 2010.

[5] L. Diening, Lindqvist P., and B. Kawohl, "Mini-Workshop: the plaplacian operator and applications," Oberwolfach Reports, vol. 10, no. 1, pp. 433-482, 2013.

[6] J. Liang and H. Yang, "Controllability of fractional integrodifferential evolution equations with nonlocal conditions," Applied Mathematics and Computation, vol. 254, pp. 20-29, 2015.

[7] K. Shah and R. A. Khan, "Study of Solution to a Toppled SYStem of Fractional Differential Equations with Integral Boundary Conditions," International Journal of Applied and Computational Mathematics, vol. 3, no. 3, pp. 2369-2388, 2017.

[8] M. Benchohra, J. R. Graef, and S. Hamani, "Existence results for boundary value problems with non-linear fractional differential equations," Applicable Analysis. An International Journal, vol. 87, no. 7, pp. 851-863, 2008.

[9] B. Ahmad and S. Sivasundaram, "On four-point nonlocal boundary value problems of nonlinear integro-differential equations of fractional order," Applied Mathematics and Computation, vol. 217, no. 2, pp. 480-487, 2010.

[10] N. Ali, K. Shah, D. Baleanu, M. Arif, and R. A. Khan, "Study of a class of arbitrary order differential equations by a coincidence degree method," Boundary Value Problems, 12 pages, 2017.
[11] H. Yang, R. P. Agarwal, and H. K. Nashine, "Coupled fixed point theorems with applications to fractional evolution equations," Advances in Difference Equations, 16 pages, 2017.

[12] B. Li and H. Gou, "Existence of solutions for impulsive fractional evolution equations with periodic boundary condition," Advances in Difference Equations, 22 pages, 2017.

[13] X. Su, "Boundary value problem for a coupled system of nonlinear fractional differential equations," Applied Mathematics Letters, vol. 22, no. 1, pp. 64-69, 2009.

[14] J. Wang, H. Xiang, and Z. Liu, "Positive solution to nonzero boundary values problem for a coupled system of nonlinear fractional differential equations," International Journal of Differential Equations, vol. 2010, Article ID 186928, 2010.

[15] W. Liu, X. Yan, and W. Qi, "Positive solutions for coupled nonlinear fractional differential equations," Journal of Applied Mathematics, vol. 2014, Article ID 790862, 7 pages, 2014.

[16] M. ur Rehman and R. A. Khan, "Existence and uniqueness of solutions for multi-point boundary value problems for fractional differential equations," Applied Mathematics Letters. An International Journal of Rapid Publication, vol. 23, no. 9, pp. 1038-1044, 2010.

[17] S. Zhang, "Positive solutions to singular boundary value problem for nonlinear fractional differential equation," Computers \& Mathematics with Applications, vol. 59, no. 3, pp. 1300-1309, 2010.

[18] B. Ahmad and J. J. Nieto, "Existence results for a coupled system of nonlinear fractional differential equations with threepoint boundary conditions," Computers \& Mathematics with Applications, vol. 58, no. 9, pp. 1838-1843, 2009.

[19] M. Iqbal, Y. Li, K. Shah, and R. A. Khan, "Application of topological degree method for solutions of coupled systems of multipoints boundary value problems of fractional order hybrid differential equations," Complexity, vol. 2017, Article ID 7676814, 9 pages, 2017.

[20] V. Gafiychuk, B. Datsko, V. Meleshko, and D. Blackmore, "Analysis of the solutions of coupled nonlinear fractional reactiondiffusion equations," Chaos, Solitons \& Fractals, vol. 41, no. 3, pp. 1095-1104, 2009.

[21] L. Gao, D. Wang, and G. Wang, "Further results on exponential stability for impulsive switched nonlinear time-delay systems with delayed impulse effects," Applied Mathematics and Computation, vol. 268, pp. 186-200, 2015.

[22] I. M. Stamova, "Mittag-Leffler stability of impulsive differential equations of fractional order," Quarterly of Applied Mathematics, vol. 73, no. 3, pp. 525-535, 2015.

[23] M. Benchohra and J. E. Lazreg, "On stability for nonlinear implicit fractional differential equations," Le Matematiche, vol. 70, no. 2, pp. 49-61, 2015.

[24] S. M. Ulam, Problems in Modern Mathematics, John Wiley and sons, New York, NY, USA, 1940.

[25] D. H. Hyers, "On the stability of the linear functional equation," Proceedings of the National Academy of Sciences of the United States of America, vol. 27, pp. 222-224, 1941.

[26] C. Urs, "Coupled fixed point theorems and applications to periodic boundary value problems," Miskolc Mathematical Notes, vol. 14, no. 1, pp. 323-333, 2013.

[27] S.-M. Jung, "On the Hyers-Ulam stability of the functional equations that have the quadratic property," Journal of Mathematical Analysis and Applications, vol. 222, no. 1, pp. 126-137, 1998. 
[28] I. A. Rus, "Ulam stabilities of ordinary differential equations in a Banach space," Carpathian Journal of Mathematics, vol. 26, no. 1, pp. 103-107, 2010.

[29] M. Gachpazan and O. Baghani, "Hyers Ulam stability of Volterra integral equation," Journal of Nonlinear Analysis and Application, pp. 19-25, 2010.

[30] Z. Ali, A. Zada, and K. Shah, "Existence and stability analysis of three point boundary value problem," International Journal of Applied and Computational Mathematic, vol. 2017, p. 14, 2017.

[31] P. Kumam, A. Ali, K. Shah, and R. . Khan, "Existence results and Hyers-Ulam stability to a class of nonlinear arbitrary order differential equations," Journal of Nonlinear Science and its Applications. JNSA, vol. 10, no. 6, pp. 2986-2997, 2017.

[32] J. Wang and X. Li, "Ulam-Hyers stability of fractional Langevin equations," Applied Mathematics and Computation, vol. 258, pp. 72-83, 2015.

[33] A. I. Perov, "On the Cauchy problem for a system of ordinary differential equations," vol. 2, pp. 115-134, 1964. 


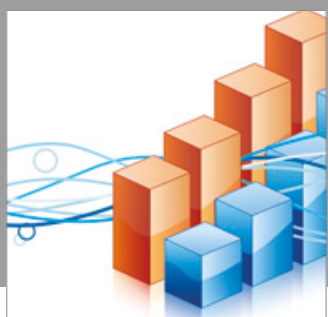

Advances in

Operations Research

vatersals

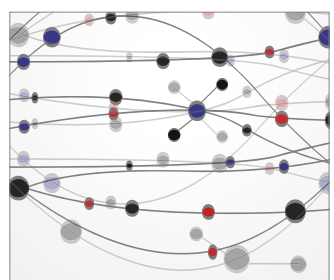

\section{The Scientific} World Journal
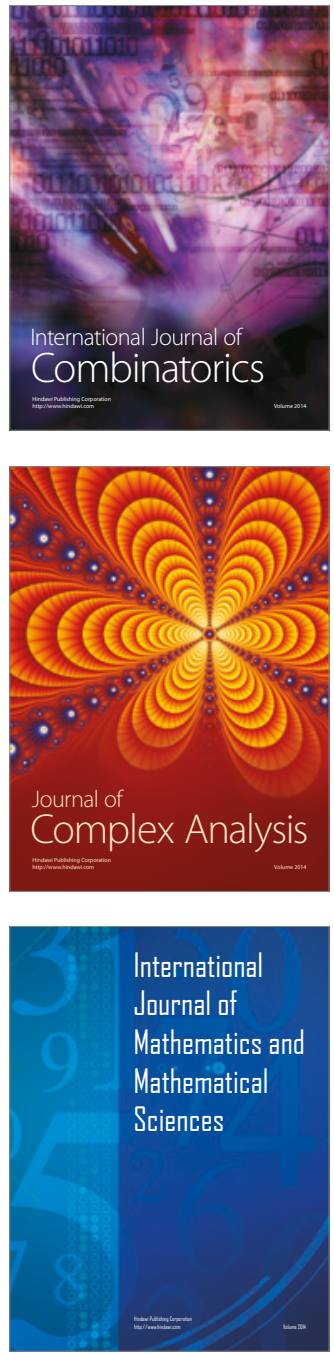
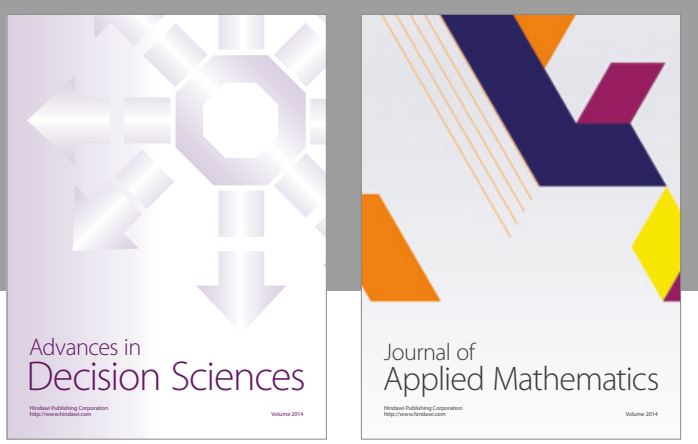

Algebra

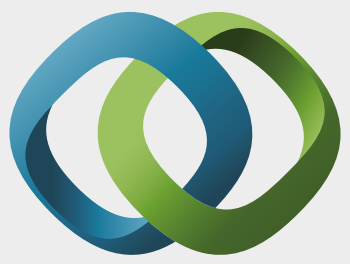

\section{Hindawi}

Submit your manuscripts at

https://www.hindawi.com
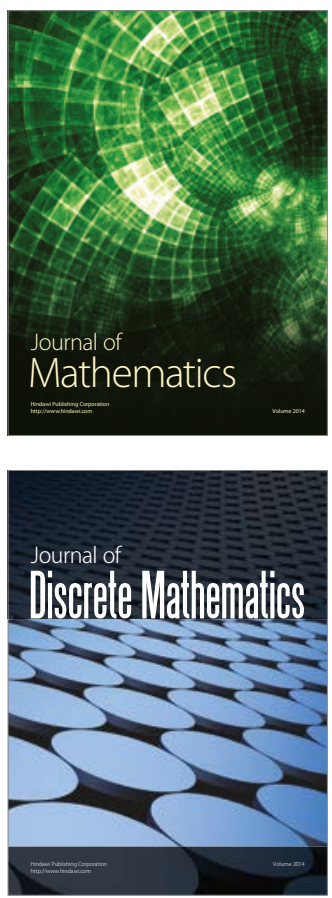

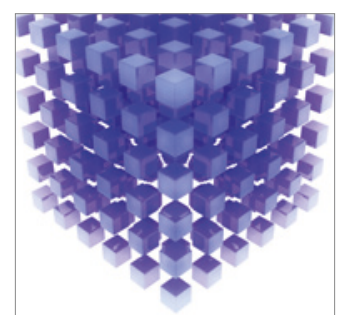

Mathematical Problems in Engineering
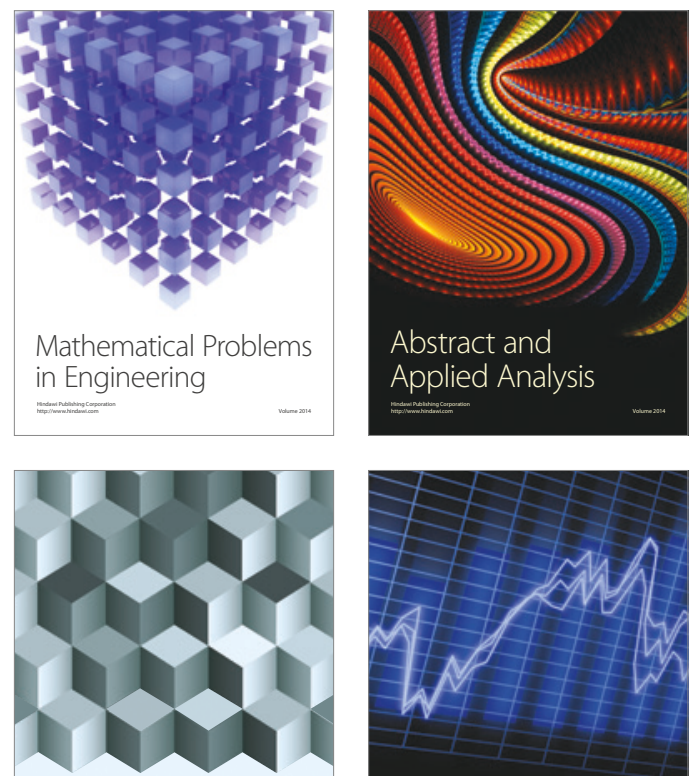

Journal of

Function Spaces

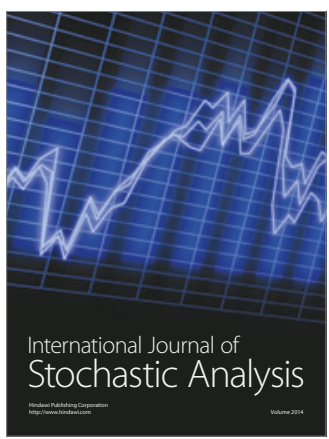

Probability and Statistics
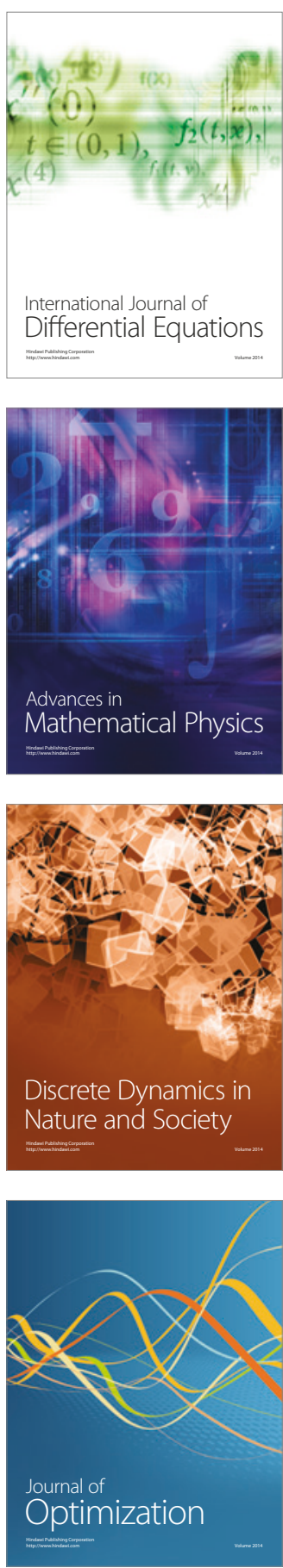